\title{
multiFLEX-LF: A Computational Approach to Quantify the Modification Stoichiometries in Label-free Proteomics Datasets
}

\author{
Pauline Hiort ${ }^{1,2}$, Christoph N. Schlaffner ${ }^{1,2,3,4}$, Judith A. Steen ${ }^{3,4,5}$, Bernhard Y. Renard ${ }^{2 \S}$, Hanno Steen ${ }^{1,5,6,7 \S^{*}}$ \\ 1 Department of Pathology, Boston Children's Hospital, Boston, MA 02115, USA, \\ 2 Data Analytics and Computational Statistics, Hasso-Plattner-Institute, Faculty of Digital Engineering, University of Potsdam, \\ Potsdam 14482, Germany, \\ 3 F. M. Kirby Neurobiology Center, Boston Children's Hospital, Boston, MA 02115, USA, \\ 4 Department of Neurology, Harvard Medical School, Boston, MA 02115, USA, \\ 5 Neurobiology Program, Boston Children's Hospital, Boston, MA 02115, USA, \\ 6 Department of Pathology, Harvard Medical School, Boston, MA 02115, USA, \\ 7 Precision Vaccines Program, Boston Children's Hospital, Boston, MA 02115, USA, \\ $\S$ B.Y.R and H.S. contributed equally \\ * Email: Hanno.Steen@childrens.harvard.edu
}

\section{Supporting Information:}

\section{Table of contents:}

Figure S1: Runtime analysis of multiFLEX-LF

Figure S2: Missing value impact on multiFLEX-LF results

Figure S3: multiFLEX-LF analysis of simulated dataset

Figure S4: Sequence logos of phospho-sites

Figure S5: Spearman correlation of mean RM score and fraction of identified modified DDA peptides per cluster

Table S1: Raw intensities of the precursors analyzed with multiFLEX-LF

Table S2: multiFLEX-LF RM scores and flat cluster IDs of the multiFLEX-LF analysis 


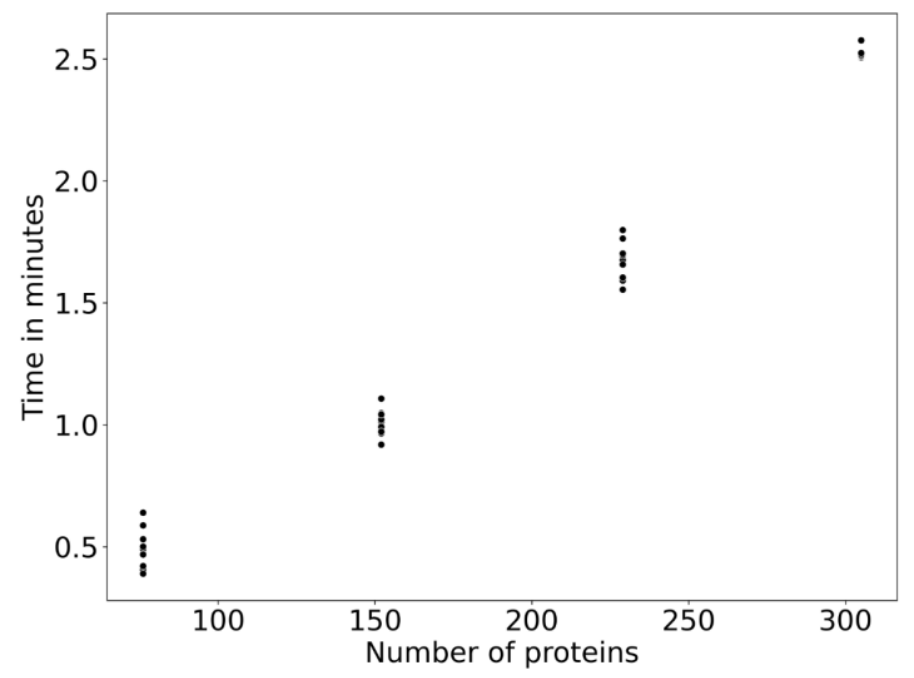

Figure S1: Runtime analysis of multiFLEX-LF. The runtime was analyzed by ten times random selection of 25, 50 and 75 percent of the proteins from our dataset and running multiFLEX-LF with the thus reduced datasets. Number of CPUs was set to 5 for these analyses using a common personal laptop with 16GB RAM and a 6-core AMD Ryzen 5 CPU. Clustering was turned on and the other settings were left at default values. 
A

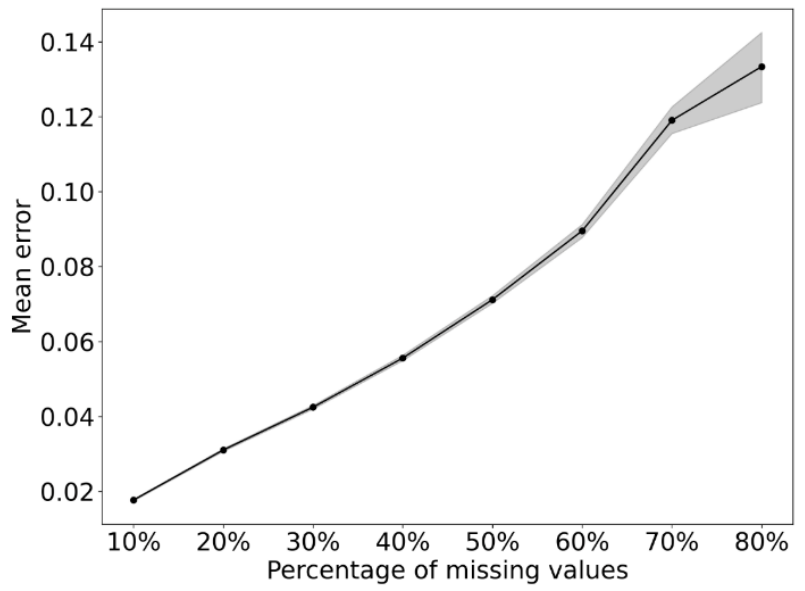

B

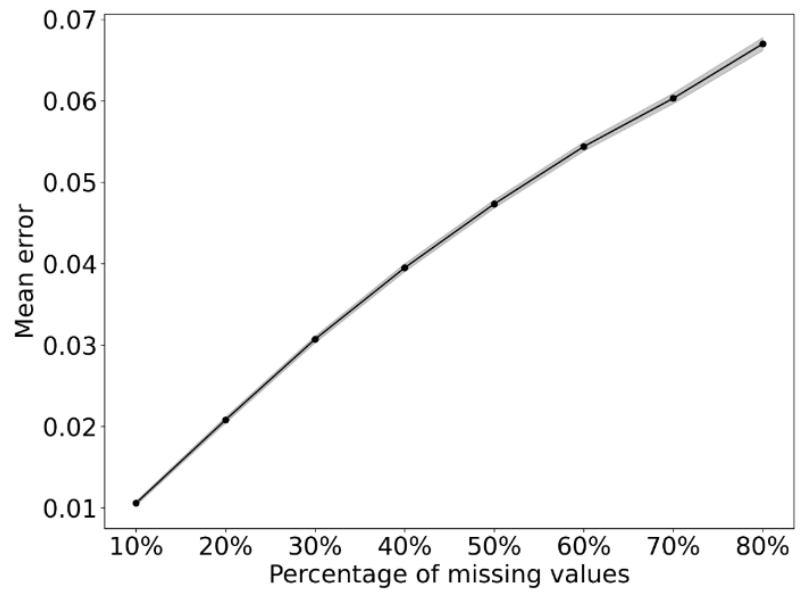

Figure S2: Missing value impact on multiFLEX-LF results. The impact of missing values was analyzed by randomly removing respectively ten times 10 to $80 \%$ of the values per sample in our dataset. Precursors with missing values in the original dataset were removed prior to this analysis. (A) Missing value impact on RM score computation. Precursor intensities were randomly removed before multiFLEX-LF analysis. RM scores of the data with randomly missing values were subtracted from the RM scores of the dataset analyzed without the generated missing intensities. As we only had one reference sample (Oh) the RM scores were all 1 for the reference therefore they were removed before computation of the difference. The figure shows the computed mean of the absolute errors with the 95\% confidence interval. (B) Missing value impact on RM scores imputation. After multiFLEX-LF computation, RM scores of the precursors were randomly removed before imputation. The error was computed by subtracting the RM scores of the imputed data from the RM scores without missing values. The RM scores of the reference sample were again removed prior to error calculation. The mean of the absolute errors is shown with the $95 \%$ confidence interval. 
A

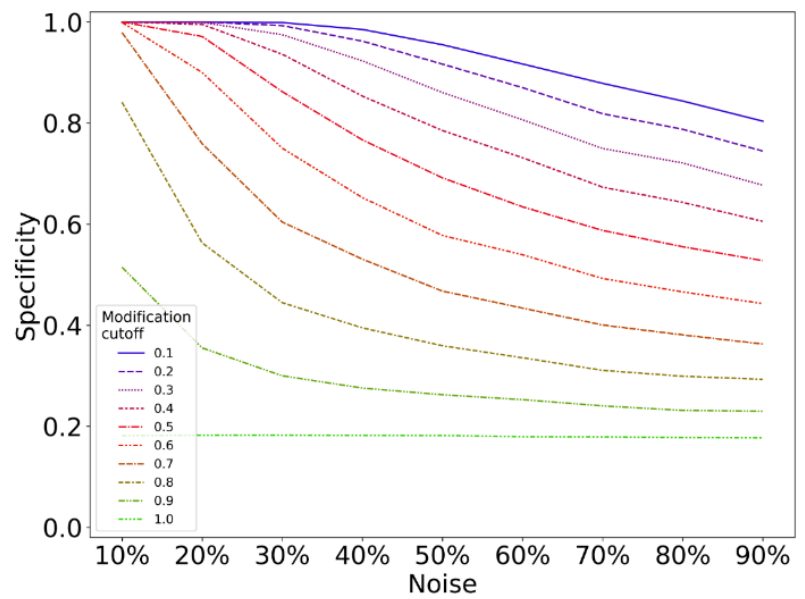

B

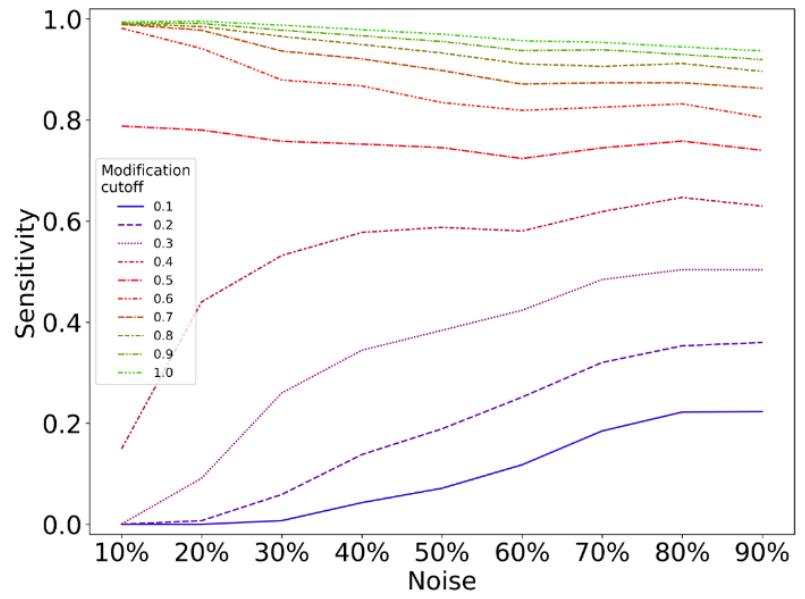

C

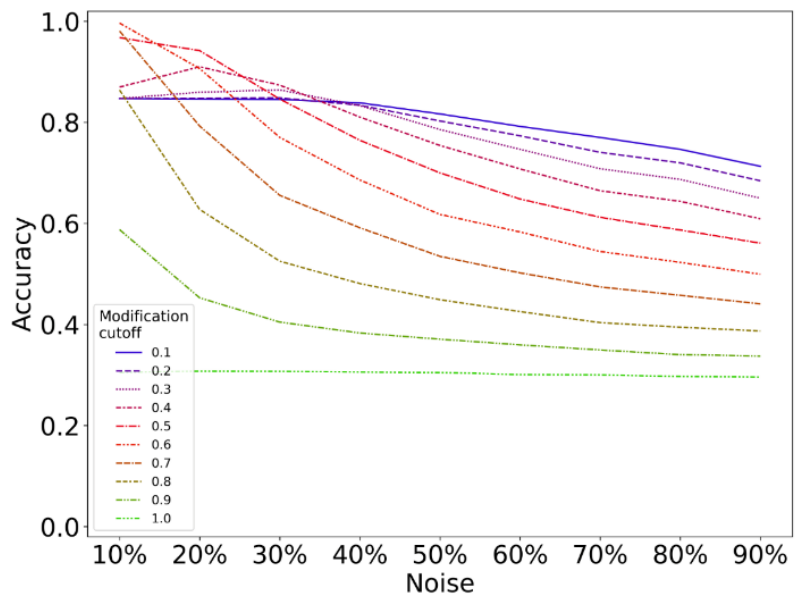

Figure S3: multifLEX-LF analysis of simulated dataset. The precursor intensities of the $0 \mathrm{~h}$ sample were used as the reference intensities for this simulation. Prior to the simulation $15 \%$ of the precursors were randomly chosen to be artificially differentially modified. For the 4,8 , and $10 \mathrm{~h}$ samples precursor intensities were drawn from a normal distribution, centered around their respective reference intensities with a standard deviation of between 10 and $90 \%$ of the reference intensity thus adding different levels of noise to the data. The intensities of the randomly chosen artificially differentially modified precursors were randomly drawn from a normal distribution centered around the $50 \%$ reduced intensity of their respective reference intensities with a standard deviation of 10 to $90 \%$ of the reduced intensity. (A) Specificity, (B) sensitivity and (C) accuracy of the RM scores with different modification cutoffs. 

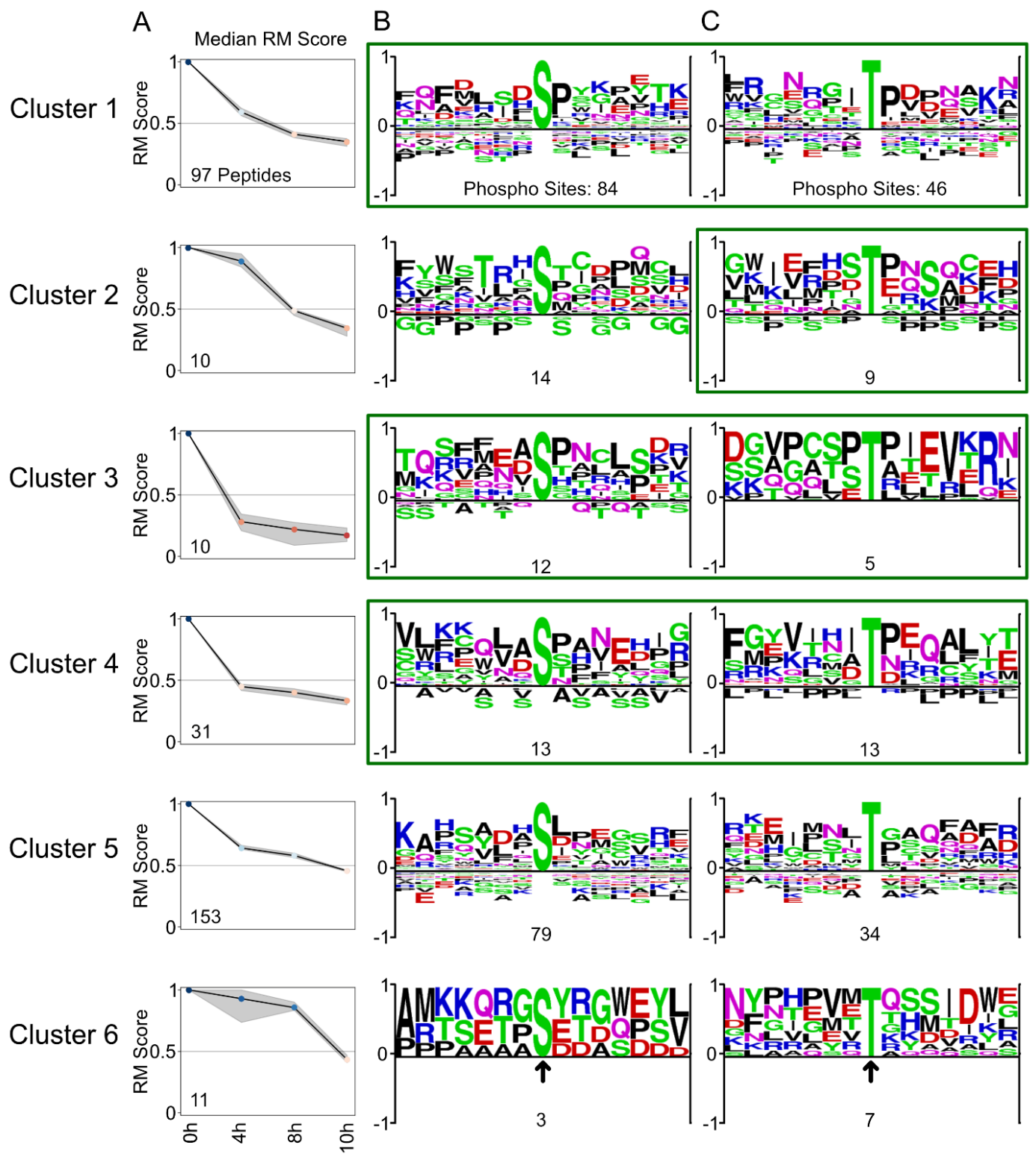

Figure S4: RM score trajectories and sequence logos of the phosphorylation sites of the clusters in Figure 2B. (A) Trajectories of the median RM scores with the $95 \%$ confidence interval around the median for each identified cluster (see also Figure 2C). (B-C) Sequence logos of the amino acid sequences around the identified known (B) serine and (C) threonine phosphorylation sites from PhosphoSitePlus ${ }^{1-3}$, respectively. Based on the known phosphorylation sites identified from PhosphoSitePlus in the precursors of the clusters, short amino acid sequences consisting of the seven preceding and following amino acids around the identified sites were acquired. These amino acid sequences of the serine and threonine phosphorylation sites were subjected to the sequence logo generator PSP LogoGenerator ${ }^{1-3}$ by PhosphoSitePlus. In the logos overrepresented amino acids are shown as positive and underrepresented amino acids as negative values. The sum of the absolute numbers of positive and negative values are kept equal to one by the PSP Production algorithm. For the logo generation, the PSP Production algorithm was selected and the background for logo generation was set to input sequences. 


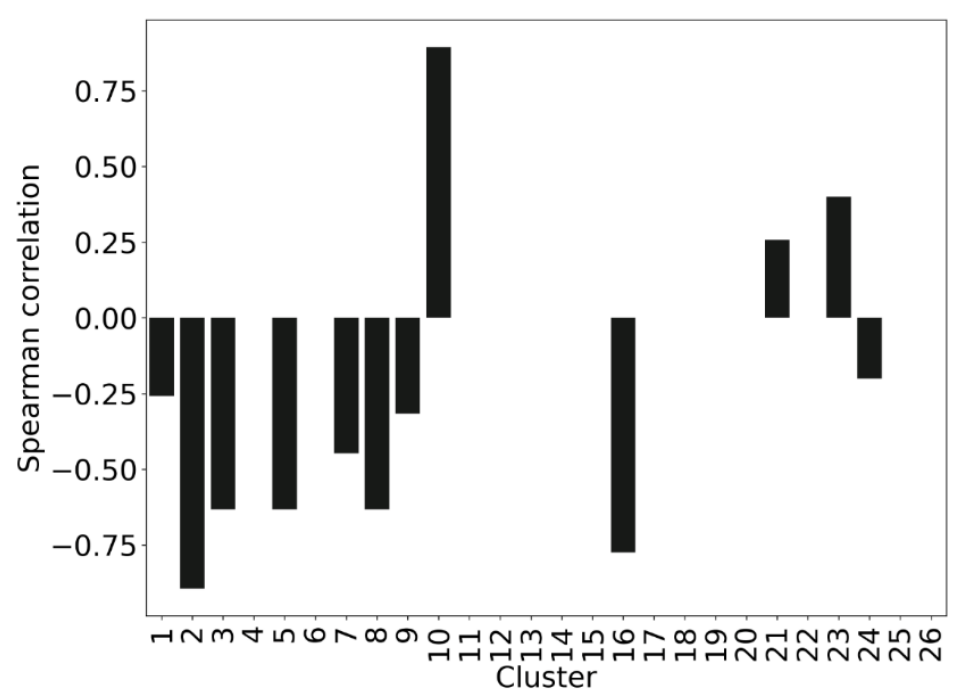

Figure S5: Spearman correlation of mean RM score and fraction of identified modified DDA peptides per cluster. Spearman correlation was computed between the mean RM score per time point for each cluster and the frequency of identified modified peptides in the DDA data per time point and cluster. The clusters are numbered according to multiFLEX-LF clustering results (Table S2). The subset of clusters that were further analyzed (Table 1) were renamed as follows: 9 to Cluster 1, 8 to Cluster 2, 6 to Cluster 3, 5 to Cluster 4, 3 to Cluster 5, 2 to Cluster 6 . Clusters which did not contain any peptides which were also found in the modified peptides of the DDA data have no correlation. The clusters which are potentially differentially modified (clusters 2 to 9 in the figure) show an anti-correlation with the number of modified DDA peptides, i.e., the lower the mean RM score/the higher the modification extent the higher the frequency of modified peptides found within the cluster. Additionally, cluster 16 shows a negative correlation, the cluster contains peptides with a RM score below 0.5 only in the $4 \mathrm{~h}$ sample. One of the peptides was found to have a modified counterpart in the DDA data also only identified in the $4 \mathrm{~h}$ sample. 


\section{References}

(1) Hornbeck, P. V.; Kornhauser, J. M.; Tkachev, S.; Zhang, B.; Skrzypek, E.; Murray, B.; Latham, V.; Sullivan, M. PhosphoSitePlus: A Comprehensive Resource for Investigating the Structure and Function of Experimentally Determined Post-Translational Modifications in Man and Mouse. Nucleic Acids Res. 2012, 40 (D1), D261-D270. https://doi.org/10.1093/nar/gkr1122.

(2) Hornbeck, P. V.; Zhang, B.; Murray, B.; Kornhauser, J. M.; Latham, V.; Skrzypek, E. PhosphoSitePlus, 2014: Mutations, PTMs and Recalibrations. Nucleic Acids Res. 2015, 43 (D1), D512-D520. https://doi.org/10.1093/nar/gku1267.

(3) Hornbeck, P. V.; Kornhauser, J. M.; Latham, V.; Murray, B.; Nandhikonda, V.; Nord, A.; Skrzypek, E.; Wheeler, T.; Zhang, B.; Gnad, F. 15 Years of PhosphoSitePlus ${ }^{\circledR}$ : Integrating Post-Translationally Modified Sites, Disease Variants and Isoforms. Nucleic Acids Res. 2019, 47 (D1), D433-D441. https://doi.org/10.1093/nar/gky1159. 